\title{
Correction to: Representation growth and rational singularities of the moduli space of local systems
}

\author{
Avraham Aizenbud $^{1}$ • Nir Avni ${ }^{2}$. \\ Roberto Rubio $^{3,4}$
}

Received: 20 October 2021 / Accepted: 4 November 2021 /

Published online: 3 February 2022

(C) Springer-Verlag GmbH Germany, part of Springer Nature 2022

Abstract We explain and correct a mistake in Section 2.6 and Appendix C of the first and second author's paper "Representation Growth and Rational Singularities of the Moduli Space of Local Systems" [1].

\section{Correction to: Invent. math. (2016) 204:245-316 https://doi.org/10.1007/s00222-015-0614-8}

We use throughout the notation and conventions of [1]. The source of the mistake is the description of the set $S_{2}$ in page 272. The elements

$$
(\{(d, d-1),(d-1, d-1)\},(d, d-1)),(\{(d-1, d-1),(d-1, d)\},(d-1, d))
$$

The third author has been funded by the Marie Sklodowska-Curie Grant Agreement No.

750885 and the Spanish R\&D Grant PID2019-109339GA-C32.

The online version of the original article can be found under https://doi.org/10.1007/s00222015-0614-8.

\footnotetext{
$凶$ Roberto Rubio

roberto.rubio@ub.edu

1 Weizmann Institute of Science, 76100 Rehovot, Israel

2 Northwestern University, Evanston, IL 60201, USA

3 Universitat de Barcelona, 08007 Barcelona, Spain

4 Universitat Autònoma de Barcelona, 08193 Barcelona, Spain
} 
are not considered. This spoils the proof of Lemma 2.40 (the blue and green subgraphs are no longer trees), which is used to prove Theorem 2.1.

\section{A straightforward correction}

The right description of $S_{2}$ is

$$
\begin{array}{r}
S_{2}=\left\{(\{(i, j),(j, l)\},(i, l)) \in I^{(2)} \times J \mid\{i, l\}=\{d-1, d\} \text { and } j=\left\lfloor\frac{i+l}{2}\right\rfloor,\right. \\
\left.\quad \text { or }\{i, l\} \neq\{d-1, d\} \text { and } j=\left\lceil\frac{i+l}{2}\right\rceil+\delta_{i, l}\right\} .
\end{array}
$$

Then, $\Gamma_{2}$ is the polygraph attached to the graph $\Gamma_{3}=(I, E)$, with

$$
\begin{array}{r}
E=\left\{\{(i, j),(j, l)\} \in I^{(2)} \mid\{i, l\}=\{d-1, d\} \text { and } j=\left\lfloor\frac{i+l}{2}\right\rfloor,\right. \\
\left.\operatorname{or}\{i, l\} \neq\{d-1, d\} \text { and } j=\left\lceil\frac{i+l}{2}\right\rceil+\delta_{i, l}\right\} .
\end{array}
$$

As for Fig. 1, 2 and 3 in [1], the two edges corresponding to (1) are missing.

Additionally, there are some mismatches concerning Fig. 2 and $\omega_{3}$ in [1]. The simplest way to make the labels of Fig. 2 match is fist to multiply $\omega_{3}$ in page 273 by 5 , obtaining

$$
w_{3}((i, j))(m)= \begin{cases}5^{|i-j|+1} & \text { if } m \equiv i-j(\bmod 3) \\ 3 \cdot 5^{|i-j|} & \text { if } m \equiv(i-j-\operatorname{sign}(i-j+1 / 2))(\bmod 3) \\ 0 & \text { if } m \equiv(i-j+\operatorname{sign}(i-j+1 / 2))(\bmod 3)\end{cases}
$$

and then consider the colouring [red is $m=0$, green is $m=2$, blue is $m=1$ ], so that we just have to swap the values of the blue and green labels along the diagonal of Fig. 2 in page 314.

Finally, the key to prove now Lemma 2.40 in the closest way to that of [1] is redefining $\omega_{3}$ at the nodes $(d-1, d-1),(d-1, d)$ and $(d, d-1)$ :

$$
\begin{array}{lll}
\omega_{3}(d-1, d-1)(0)=3 \cdot 5^{1}, & \omega_{3}(d-1, d)(0)=5^{2}, & \omega_{3}(d, d-1)(0)=3 \cdot 5^{0}, \\
\omega_{3}(d-1, d-1)(2)=3 \cdot 5^{1}, & \omega_{3}(d-1, d)(2)=4 \cdot 5^{1}, & \omega_{3}(d, d-1)(2)=0, \\
\omega_{3}(d-1, d-1)(1)=0, & \omega_{3}(d-1, d)(1)=0, & \omega_{3}(d, d-1)(1)=5^{2},
\end{array}
$$

so that the resulting diagram for $d=6$ (Fig. 2 in [1]) is given by Image 1 .

With the redefinition of $\omega_{3}$ above, we only need to add to the proof of Lemma 2.40 in [1] an analysis of the edges around $(d-1, d-1)$. They look, 


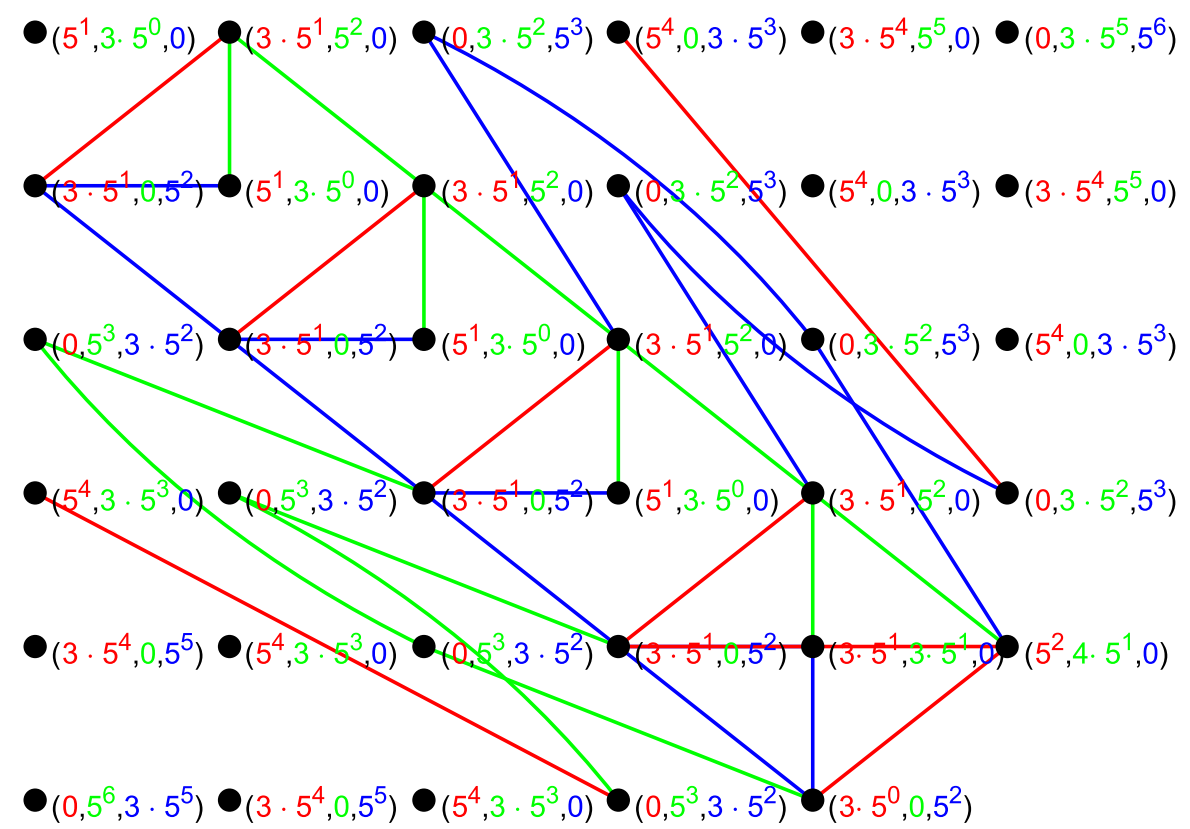

Image 1 Graph $\Gamma_{3}$ with the weights $\omega_{3}$ for the case $d=6$

for $d \geq 3$, like the ones in Image 1 . We thus have forests with maximal degree $\leq 3$, as we need. Finally, the cases $d=1,2$ are straigthforward.

\section{An alternative solution}

We indicate here how to get an alternative solution that, although requires more changes, would keep better the original intuition for the proof.

At the beginning of Section 2.6 in [1], recall that $L=\{1, \ldots, d\}$. Stay with $J=L \times L \backslash\{(d, d)\}$ and replace $I$ by $I=L \times L \backslash\{(1,1)\}$. This entails changes in $S_{j}$ and $\Gamma_{j}$, which we omit here for the sake of brevity. With the definition of $\omega_{3}$ as in (2) (without any redefinition) and the same conventions for the colours as described in Sect. 1, the corresponding $\Gamma_{3}$ for $d=6$ is given by Image 2.

The general proof then follows along the same lines as the original one. 


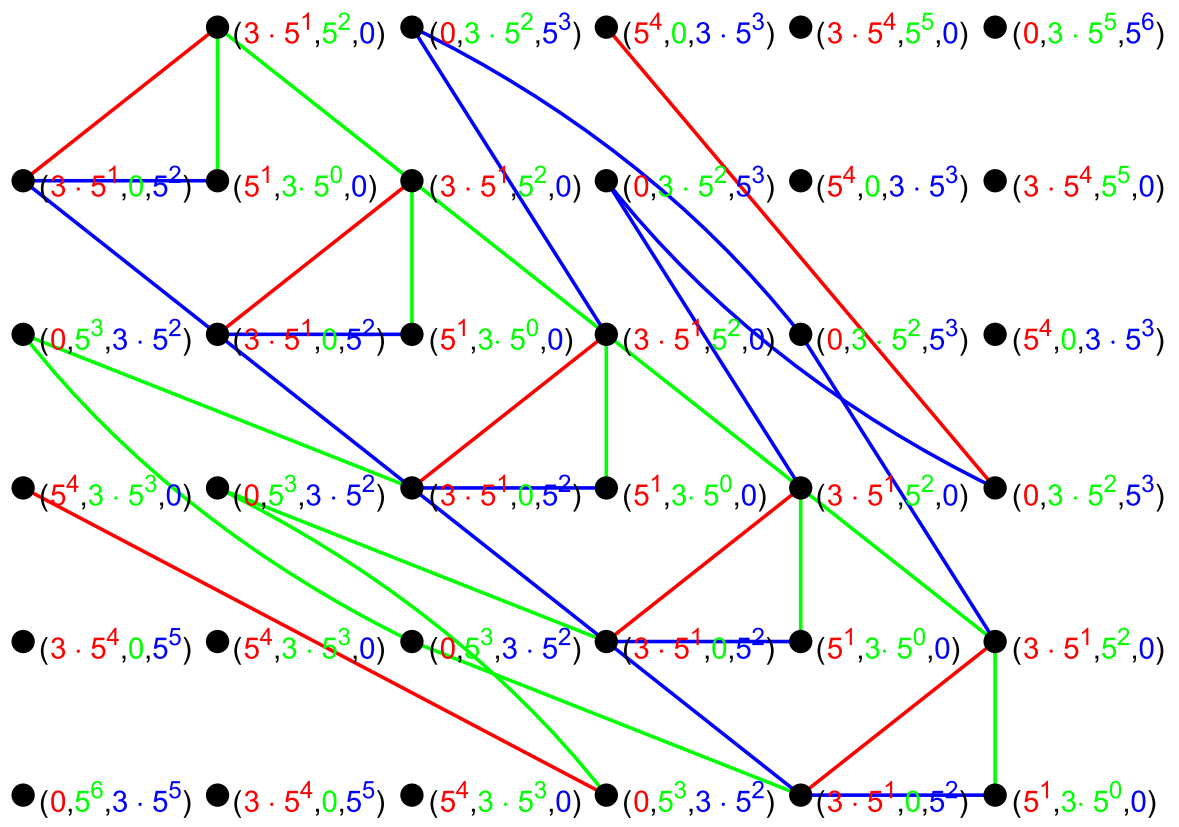

Image 2 Graph $\Gamma_{3}$ with $I \neq J$ for the case $d=6$

Acknowledgements The third author thanks S. Carmeli for introducing the basics of [1] to him, and the Weizmann Institute of Science for its hospitality.

\section{References}

1. Aizenbud, A., Avni, N.: Representation growth and rational singularities of the moduli space of local systems. Invent. Math. 204(1), 245-316 (2016)

Publisher's Note Springer Nature remains neutral with regard to jurisdictional claims in published maps and institutional affiliations. 